\title{
Global Optimization for Solving Linear Multiplicative Programming Based on a New Linearization Method
}

\author{
Chun-Feng Wang ${ }^{1,2}$ and Yan-Qin Bai ${ }^{1}$ \\ ${ }^{1}$ Department of Mathematics, College of Sciences, Shanghai University, Shanghai 200444, China \\ ${ }^{2}$ Department of Mathematics, Henan Normal University, Xinxiang 453007, China \\ Correspondence should be addressed to Chun-Feng Wang; wangchunfeng09@126.com
}

Received 22 April 2016; Revised 22 July 2016; Accepted 31 July 2016

Academic Editor: Fabrizio Riguzzi

Copyright (c) 2016 C.-F. Wang and Y.-Q. Bai. This is an open access article distributed under the Creative Commons Attribution License, which permits unrestricted use, distribution, and reproduction in any medium, provided the original work is properly cited.

This paper presents a new global optimization algorithm for solving a class of linear multiplicative programming (LMP) problem. First, a new linear relaxation technique is proposed. Then, to improve the convergence speed of our algorithm, two pruning techniques are presented. Finally, a branch and bound algorithm is developed for solving the LMP problem. The convergence of this algorithm is proved, and some experiments are reported to illustrate the feasibility and efficiency of this algorithm.

\section{Introduction}

Consider the following linear multiplicative programming (LMP) problem:

LMP:

$$
\begin{array}{cl}
v=\min & \phi(x)=\sum_{i=1}^{p}\left(c_{i}^{T} x+d_{i}\right)\left(e_{i}^{T} x+f_{i}\right) \\
\text { s.t. } \quad x \in D=\left\{x \in R^{n} \mid A x \leq b\right\},
\end{array}
$$

where $p \geq 2, c_{i}^{T}=\left(c_{i 1}, c_{i 2}, \ldots, c_{i n}\right), e_{i}^{T}=\left(e_{i 1}, e_{i 2}, \ldots, e_{i n}\right) \epsilon$ $R^{n}, d_{i}, f_{i} \in R, i=1, \ldots, p, A=\left(a_{i j}\right)_{m \times n} \in R^{m \times n}$ is a matrix, $b=\left(b_{i}\right)_{m \times 1} \in R^{m}$ is a vector, and $D \subseteq R^{n}$ is nonempty and bounded.

As a special case of nonconvex programming problem, the problem LMP has been paid more attention since the 1990s. There are two reasons. The first one is that, from a practical point of view, LMP problem appears in a wide variety of practical applications, such as financial optimization [1], data mining/pattern recognition [2], plant layout design [3], VLISI chip design [4], and robust optimization [5]. The second one is that, from a research point of view, LMP is N-hard; that is, it usually possesses multiple local optimal solutions that are not globally optimal. So, it is hard to find its global optimal solution, and it is necessary to put forward good methods.
In the past few decades, for all $x \in D$, under the assumption that $c_{i}^{T}+d_{i}>0, e_{i}^{T} x+f_{i}>0$, a number of practical algorithms have been proposed for globally solving problem LMP. These methods can be classified into parameterization based methods $[6,7]$, branch-and-bound methods [8-10], decomposition method [11], cutting plane method [12], and so on.

The purpose of this paper is to present an effective method for globally solving problem LMP. Compared with other algorithms, the main features of this algorithm are (1) by using the special structure of LMP, a new linear relaxation technique is presented, which can be used to construct the linear relaxation programming (LRP) problem; (2) two pruning techniques are presented, which can be used to improve the convergence speed of the proposed algorithm; (3) the problem investigated in this paper has a more general form than those in [6-12]; it does not require $c_{i}^{T} x+d_{i}>0$ and $e_{i}^{T} x+f_{i}>0$; (4) numerical results and comparison with methods $[8,13-22]$ show that our algorithm works as well as or better than those methods.

This paper is organized as follows. In Section 2, the new linear relaxation programming (LRP) problem for LMP problem is proposed, which provides a lower bound for the optimal value of LMP. In order to improve the convergence speed of our algorithm, two pruning techniques are presented in Section 3. In Section 4, the global optimization algorithm 
is given, and the convergence of the algorithm is proved. Numerical experiments are carried out to show the feasibility and efficiency of our algorithm in Section 5.

\section{Linear Relaxation Programming (LRP)}

To solve problem LMP, the principal task is the construction of lower bound for this problem and its partitioned subproblems. A lower bound of LMP problem and its partitioned subproblems can be obtained by solving a linear relaxation programming problem. For generating this linear relaxation, the strategy proposed by this paper is to underestimate the objective function $\phi(x)$ with a linear function. All the details of this procedure will be given in the following.

First, we solve $2 n$ linear programming problems: $l_{j}^{0}=$ $\min _{x \in D} x_{j}, u_{j}^{0}=\max _{x \in D} x_{j}, j=1, \ldots, n$, and construct a rectangle $H^{0}=\left\{x \in R^{n} \mid l_{j}^{0} \leq x_{j} \leq u_{j}^{0}, j=1, \ldots, n\right\}$. Then, the LMP problem can be rewritten as the following form:

LMP:

$$
\begin{array}{ll}
v=\min & \phi(x)=\sum_{i=1}^{p}\left(c_{i}^{T} x+d_{i}\right)\left(e_{i}^{T} x+f_{i}\right) \\
\text { s.t. } & x \in D=\left\{x \in R^{n} \mid A x \leq b\right\}, \\
& H^{0}=\left\{x \mid l_{j}^{0} \leq x_{j} \leq u_{j}^{0}, j=1, \ldots, n\right\} .
\end{array}
$$

Let $H=[l, u]$ be the initial rectangle $H^{0}$ or some subrectangle of $H^{0}$ that is generated by the proposed algorithm. Next, we will show how to construct the linear relaxation programming problem LRP for LMP.

Towards this end, for $i=1, \ldots, p$, compute

$$
\begin{aligned}
& \underline{\xi}_{i}=\sum_{j=1}^{n} \min \left\{c_{i j} l_{j}, c_{i j} u_{j}\right\}+d_{i}, \\
& \bar{\xi}_{i}=\sum_{j=1}^{n} \max \left\{c_{i j} l_{j}, c_{i j} u_{j}\right\}+d_{i}, \\
& \underline{\eta}_{i}=\sum_{j=1}^{n} \min \left\{e_{i j} l_{j}, e_{i j} u_{j}\right\}+f_{i}, \\
& \bar{\eta}_{i}=\sum_{j=1}^{n} \max \left\{e_{i j} l_{j}, e_{i j} u_{j}\right\}+f_{i},
\end{aligned}
$$

and consider the product term $\left(c_{i}^{T} x+d_{i}\right)\left(e_{i}^{T} x+f_{i}\right)$ in $\phi(x)$.

Since $c_{i}^{T} x+d_{i}-\underline{\xi}_{i} \geq 0, e_{i}^{T} x+f_{i}-\underline{\eta}_{i} \geq 0$, we have

$$
\left(c_{i}^{T} x+d_{i}-\underline{\xi}_{i}\right)\left(e_{i}^{T} x+f_{i}-\underline{\eta}_{i}\right) \geq 0
$$

that is,

$$
\begin{aligned}
\left(c_{i}^{T} x\right. & \left.+d_{i}\right)\left(e_{i}^{T} x+f_{i}\right)-\underline{\eta}_{i}\left(c_{i}^{T} x+d_{i}\right)-\underline{\xi}_{i}\left(e_{i}^{T} x+f_{i}\right) \\
& +\underline{\xi}_{i} \underline{\eta}_{i} \geq 0 .
\end{aligned}
$$

Furthermore, we have

$$
\begin{aligned}
\left(c_{i}^{T} x+d_{i}\right)\left(e_{i}^{T} x+f_{i}\right) \geq & \underline{\eta}_{i}\left(c_{i}^{T} x+d_{i}\right)+\underline{\xi}_{i}\left(e_{i}^{T} x+f_{i}\right) \\
& -\underline{\xi}_{i} \underline{\eta}_{i} .
\end{aligned}
$$
have

In addition, since $c_{i}^{T} x+d_{i}-\underline{\xi}_{i} \geq 0, e_{i}^{T} x+f_{i}-\bar{\eta}_{i} \leq 0$, we

$$
\left(c_{i}^{T} x+d_{i}-\underline{\xi}_{i}\right)\left(e_{i}^{T} x+f_{i}-\bar{\eta}_{i}\right) \leq 0 .
$$

Furthermore, we can obtain

$$
\begin{aligned}
\left(c_{i}^{T} x+d_{i}\right)\left(e_{i}^{T} x+f_{i}\right) \leq & \bar{\eta}_{i}\left(c_{i}^{T} x+d_{i}\right)+\underline{\xi}_{i}\left(e_{i}^{T} x+f_{i}\right) \\
& -\underline{\xi}_{i} \bar{\eta}_{i} .
\end{aligned}
$$

From (6) and (8), we have the following relations:

$$
\begin{aligned}
\phi(x) & =\sum_{i=1}^{p}\left(c_{i}^{T} x+d_{i}\right)\left(e_{i}^{T} x+f_{i}\right) \\
& \geq \sum_{i=1}^{p}\left[\underline{\eta}_{i}\left(c_{i}^{T} x+d_{i}\right)+\underline{\xi}_{i}\left(e_{i}^{T} x+f_{i}\right)-\underline{\xi}_{i} \underline{\eta}_{i}\right] \\
& =\phi^{l}(x), \\
\phi(x) & =\sum_{i=1}^{p}\left(c_{i}^{T} x+d_{i}\right)\left(e_{i}^{T} x+f_{i}\right) \\
& \leq \sum_{i=1}^{p}\left[\bar{\eta}_{i}\left(c_{i}^{T} x+d_{i}\right)+\underline{\xi}_{i}\left(e_{i}^{T} x+f_{i}\right)-\underline{\xi}_{i} \bar{\eta}_{i}\right] \\
& =\phi^{u}(x) .
\end{aligned}
$$

Based on the above discussion, the linear relaxation programming (LRP) problem can be established as follows, which provides a lower bound for the optimal value of LMP problem over $H$ :

LRP:

$$
\begin{array}{ll}
\min & \phi^{l}(x) \\
\text { s.t. } & A x \leq b, \\
& x \in H .
\end{array}
$$

Theorem 1. For all $x \in H$, let $\Delta x=u-l$ and consider the functions $\phi^{l}(x), \phi(x)$, and $\phi^{u}(x)$. Then, one has $\lim _{\Delta x \rightarrow 0}\left(\phi(x)-\phi^{l}(x)\right)=\lim _{\Delta x \rightarrow 0}\left(\phi^{u}(x)-\phi(x)\right)=0$.

Proof. We first prove $\lim _{\Delta x \rightarrow 0}\left(\phi(x)-\phi^{l}(x)\right)=0$. By the definitions $\phi(x)$ and $\phi^{l}(x)$, we have

$$
\begin{aligned}
& \left|\phi(x)-\phi^{l}(x)\right|=\mid \sum_{i=1}^{p}\left(c_{i}^{T} x+d_{i}\right)\left(e_{i}^{T} x+f_{i}\right) \\
& -\sum_{i=1}^{p}\left[\underline{\eta}_{i}\left(c_{i}^{T} x+d_{i}\right)+\underline{\xi}_{i}\left(e_{i}^{T} x+f_{i}\right)-\underline{\xi}_{i} \underline{\eta}_{i}\right] \mid
\end{aligned}
$$




$$
\begin{aligned}
& \leq\left|\sum_{i=1}^{p}\left[\left(c_{i}^{T} x+d_{i}\right)\left(e_{i}^{T} x+f_{i}\right)-\underline{\eta}_{i}\left(c_{i}^{T} x+d_{i}\right)\right]\right| \\
& +\left|\sum_{i=1}^{p}\left[\underline{\xi}_{i}\left(e_{i}^{T} x+f_{i}\right)-\underline{\xi}_{i} \underline{\eta}_{i}\right]\right| \\
& \leq \sum_{i=1}^{p}\left|c_{i}^{T} x+d_{i}\right|\left|\left(e_{i}^{T} x+f_{i}\right)-\underline{\eta}_{i}\right| \\
& +\sum_{i=1}^{p}\left|\underline{\xi}_{i}\right|\left|e_{i}^{T} x+f_{i}-\underline{\eta}_{i}\right| .
\end{aligned}
$$

Sice $D$ is nonempty and bounded, there exists $M_{i}$ such that $M_{i}=\max _{x \in D}\left|c_{i}^{T} x+d_{i}\right|$. From the above inequality, we have

$$
\left|\phi(x)-\phi^{l}(x)\right| \leq \sum_{i=1}^{p} M_{i}\left|\bar{\eta}_{i}-\underline{\eta}_{i}\right|+\sum_{i=1}^{p}\left|\underline{\xi}_{i}\right|\left|\bar{\eta}_{i}-\underline{\eta}_{i}\right| .
$$

By the definitions of $\underline{\eta}_{i}$ and $\bar{\eta}_{i}$, we know that $\Delta s=\bar{\eta}_{i}-\eta_{i} \rightarrow 0$ as $\Delta x \rightarrow 0$. Combining (12), we have $\lim _{\Delta x \rightarrow 0}\left(\phi(x)-\phi^{\bar{l}}(x)\right)=$ 0 .

Similarly, we can prove $\lim _{\Delta x \rightarrow 0}\left(\phi^{u}(x)-\phi(x)\right)=0$, and the proof is complete.

Theorem 1 implies that $\phi^{l}(x)$ and $\phi^{u}(x)$ will approximate the function $\phi(x)$ as $\Delta x \rightarrow 0$.

\section{Pruning Technique}

To improve the convergence speed of this algorithm, we present two pruning techniques, which can be used to eliminate the region in which the global optimal solution of LMP problem does not exist.

Assume that UB and LB are the current known upper bound and lower bound of the optimal value $v$ of the problem LMP. Let

$$
\begin{aligned}
\alpha_{j} & =\sum_{i=1}^{p}\left(\underline{\eta}_{i} c_{i j}+\underline{\xi}_{i} e_{i j}\right), \quad j=1, \ldots, n, \\
\Lambda_{1} & =\sum_{i=1}^{p}\left(\underline{\eta}_{i} d_{i}+\underline{\xi} f_{i}-\underline{\eta}_{i} \underline{\xi}\right), \\
\gamma_{k} & =\mathrm{UB}-\sum_{j=1, j \neq k}^{n} \min \left\{\alpha_{j} l_{j}, \alpha_{j} u_{j}\right\}-\Lambda_{1}, \\
\beta_{j} & =\sum_{i=1}^{p}\left(\bar{\eta}_{i} c_{i j}+\underline{\xi}_{i} e_{i j}\right), \quad j=1, \ldots, n, \quad k=1, \ldots, n, \\
\Lambda_{2} & =\sum_{i=1}^{p}\left(\bar{\eta}_{i} d_{i}+\underline{\xi} f_{i}-\bar{\eta}_{i} \underline{\xi}\right),
\end{aligned}
$$

$$
\begin{aligned}
\rho_{k}=\mathrm{LB}-\sum_{j=1, j \neq k}^{n} \max \left\{\beta_{j} l_{j}, \beta_{j} u_{j}\right\}-\Lambda_{2}, & \\
& k=1, \ldots, n .
\end{aligned}
$$

The pruning techniques are derived as in the following theorems.

Theorem 2. For any subrectangle $H \subseteq H^{0}$ with $H_{j}=\left[l_{j}, u_{j}\right]$, if there exists some index $k \in\{1,2, \ldots, n\}$ such that $\alpha_{k}>0$ and $\gamma_{k}<\alpha_{k} u_{k}$, then there is no globally optimal solution of LMP problem over $H^{1}$; if $\alpha_{k}<0$ and $\gamma_{k}<\alpha_{k} l_{k}$, for some $k$, then there is no globally optimal solution of LMP problem over $H^{2}$, where

$$
\begin{aligned}
& H^{1}=\left(H_{j}^{1}\right)_{n \times 1} \subseteq H, \\
& \text { with } H_{j}^{1}= \begin{cases}H_{j}, & j \neq k, \\
\left(\frac{\gamma_{k}}{\alpha_{k}}, u_{k}\right] \cap H_{k}, & j=k,\end{cases} \\
& H^{2}=\left(H_{j}^{2}\right)_{n \times 1} \subseteq H, \\
& \text { with } H_{j}^{2}= \begin{cases}H_{j}, & j \neq k, \\
{\left[l_{k}, \frac{\gamma_{k}}{\alpha_{k}}\right) \cap H_{k},} & j=k .\end{cases}
\end{aligned}
$$

Proof. For all $x \in H^{1}$, we first show that $\phi(x)>$ UB. Consider the $k$ th component $x_{k}$ of $x$. Since $x_{k} \in\left(\gamma_{k} / \alpha_{k}, u_{k}\right]$, we can obtain that

$$
\frac{\gamma_{k}}{\alpha_{k}}<x_{k} \leq u_{k}
$$

From $\alpha_{k}>0$, we have $\gamma_{k}<\alpha_{k} x_{k}$. For all $x \in H^{1}$, by the above inequality and the definition of $\gamma_{k}$, it implies that

$$
\mathrm{UB}-\sum_{j=1, j \neq k}^{n} \min \left\{\alpha_{j} l_{j}, \alpha_{j} u_{j}\right\}-\Lambda_{1}<\alpha_{k} x_{k} ;
$$

that is,

$$
\begin{aligned}
\mathrm{UB} & <\sum_{j=1, j \neq k}^{n} \min \left\{\alpha_{j} l_{j}, \alpha_{j} u_{j}\right\}+\alpha_{k} x_{k}+\Lambda_{1} \\
& \leq \sum_{j=1}^{n} \alpha_{j} x_{j}+\Lambda_{1}=\phi^{l}(x) .
\end{aligned}
$$

Thus, for all $x \in H^{1}$, we have $\phi(x) \geq \phi^{l}(x)>\mathrm{UB} \geq v$; that is, for all $x \in H^{1}, \phi(x)$ is always greater than the optimal value $v$ of the problem LMP. Therefore, there cannot exist globally optimal solution of LMP problem over $H^{1}$.

Similarly, for all $x \in H^{2}$, if there exists some $k$ such that $\alpha_{k}<0$ and $\gamma_{k}<\alpha_{k} l_{k}$, it can be derived that there is no globally optimal solution of LMP problem over $H^{2}$. 
Theorem 3. For any subrectangle $H \subseteq H^{0}$ with $H_{j}=\left[l_{j}, u_{j}\right]$, if there exists some index $k \in\{1,2, \ldots, n\}$ such that $\beta_{k}>0$ and $\rho_{k}>\beta_{k} l_{k}$, then there is no globally optimal solution of LMP problem over $H^{3}$; if $\beta_{k}<0$ and $\rho_{k}>\beta_{k} u_{k}$, for some $k$, then there is no globally optimal solution of LMP problem over $\mathrm{H}^{4}$, where

$$
\begin{aligned}
& H^{3}=\left(H_{j}^{3}\right)_{n \times 1} \subseteq H, \\
& \text { with } H_{j}^{3}= \begin{cases}H_{j}, & j \neq k, \\
{\left[l_{k}, \frac{\rho_{k}}{\beta_{k}}\right) \cap H_{k},} & j=k,\end{cases} \\
& H^{4}=\left(H_{j}^{4}\right)_{n \times 1} \subseteq H, \\
& \text { with } H_{j}^{4}= \begin{cases}H_{j}, & j \neq k, \\
\left(\frac{\rho_{k}}{\beta_{k}}, u_{k}\right] \cap H_{k}, & j=k .\end{cases}
\end{aligned}
$$

Proof. First, we show that, for all $x \in H^{3}, \phi(x)<$ LB. Consider the $k$ th component $x_{k}$ of $x$. By the assumption and the definitions of $\beta_{k}$ and $\rho_{k}$, we have

$$
l_{k} \leq x_{k}<\frac{\rho_{k}}{\beta_{k}} \text {. }
$$

Note that since $\beta_{k}>0$, we have $\rho_{k}>\beta_{k} x_{k}$. For all $x \in H^{3}$, by the above inequality and the definition of $\rho_{k}$, it implies that

$$
\begin{aligned}
\mathrm{LB} & >\sum_{j=1, j \neq k}^{n} \max \left\{\beta_{j} l_{j}, \beta_{j} u_{j}\right\}+\beta_{k} x_{k}+\Lambda_{2} \\
& \geq \sum_{j=1}^{n} \beta_{j} x_{j}+\Lambda_{2}=\Phi^{u}(x) \geq \phi(x) .
\end{aligned}
$$

Thus, for all $x \in H^{3}$, we have $v \geq \operatorname{LB}>\phi(x)$. Therefore, there cannot exist globally optimal solution of LMP problem over $H^{3}$.

For all $x \in H^{4}$, if there exists some $k$ such that $\beta_{k}<0$ and $\rho_{k}>\beta_{k} u_{k}$, from arguments similar to the above, it can be derived that there is no globally optimal solution of LMP problem over $H^{4}$.

\section{Algorithm and Its Convergence}

Based on the previous results, this section presents the branch and bound algorithm and gives its convergence.

4.1. Branching Rule. In branch and bound algorithm, branch rule is a critical element in guaranteeing convergence. This paper chooses a simple and standard bisection rule, which is sufficient to ensure convergence since it drives the intervals shrinking to a singleton for all the variables along any infinite branch of the branch and bound tree.

Consider any node subproblem identified by rectangle $H=\left\{x \in R^{n} \mid l_{j} \leq x_{j} \leq u_{j}, j=1, \ldots, n\right\} \subseteq H^{0}$. The branching rule is as follows: (i) let $k=\operatorname{argmax}\left\{u_{j}-l_{j} \mid j=1, \ldots, n\right\}$;

(ii) let $\tau=\left(l_{k}+u_{k}\right) / 2$;

(iii) let

$$
\begin{aligned}
& H^{1}=\left\{x \in R^{n} \mid l_{j} \leq x_{j} \leq u_{j}, j \neq k, l_{k} \leq x_{k} \leq \tau\right\}, \\
& H^{2}=\left\{x \in R^{n} \mid l_{j} \leq x_{j} \leq u_{j}, j \neq k, \tau \leq x_{k} \leq u_{k}\right\} .
\end{aligned}
$$

Through using this branching rule, the rectangle $H$ is partitioned into two subrectangles $H^{1}$ and $H^{2}$.

4.2. Branch and Bound Algorithm. From the above discussion, the branch and bound algorithm for globally solving LMP problem is summarized as follows.

Let $\operatorname{LB}\left(H^{k}\right)$ be the optimal function value of LRP over the subrectangle $H=H^{k}$ and $x^{k}=x\left(H^{k}\right)$ be an element of the corresponding argmin.

\section{Algorithm Statement}

Step 1. Choose $\epsilon \geq 0$. Find an optimal solution $x^{0}=x\left(H^{0}\right)$ and the optimal value $\operatorname{LB}\left(H^{0}\right)$ for problem LRP with $H=H^{0}$. Set $\mathrm{LB}_{0}=\mathrm{LB}\left(H^{0}\right)$, and $\mathrm{UB}_{0}=\phi\left(x^{0}\right)$. If $\mathrm{UB}_{0}-\mathrm{LB}_{0} \leq \epsilon$, then stop: $x^{0}$ is an $\epsilon$-optimal solution of problem LMP. Otherwise, set $Q_{0}=\left\{H^{0}\right\}, k=1$, and go to Step 2 .

Step 2. Set $\mathrm{UB}_{k}=\mathrm{UB}_{k-1}$. Subdivide $H^{k-1}$ into two subrectangles via the branching rule, and denote the set of new partition rectangles as $\bar{H}^{k}$.

Step 3. For each new rectangle $H \in \bar{H}^{k}$, utilizing the pruning techniques of Theorems 2 and 3 to prune rectangle $H$. For $i=1, \ldots, m$, if there exists some $i$ such that $\sum_{j=1}^{n} \min \left\{a_{i j} l_{j}, a_{i j} u_{j}\right\} \geq b_{i}$ over rectangle $H$, then remove the rectangle $H$ from $\bar{H}^{k}$; that is, $\bar{H}^{k}=\bar{H}^{k} \backslash H$.

Step 4. If $\bar{H}^{k} \neq \emptyset$, solve LRP to obtain $\operatorname{LB}(H)$ and $x(H)$ for each $H \in \bar{H}^{k}$. If $\mathrm{LB}(H)>\mathrm{UB}_{k}$, set $\bar{H}^{k}=\bar{H}^{k} \backslash H$. Otherwise, let $\mathrm{UB}_{k}=\min \left\{\mathrm{UB}_{k}, \phi(x(H))\right\}$. If $\mathrm{UB}_{k}=\phi(x(H))$, set $x^{k}=$ $x(H)$.

Step 5. Set

$$
Q_{k}=\left\{Q_{k-1} \backslash H^{k-1}\right\} \cup \bar{H}^{k}
$$

Step 6. Set $\mathrm{LB}_{k}=\min \left\{\mathrm{LB}(H) \mid H \in \mathrm{Q}_{k}\right\}$. Let $H^{k}$ be the subrectangle which satisfies that $\mathrm{LB}_{k}=\mathrm{LB}\left(H^{k}\right)$. If $\mathrm{UB}_{k}-$ $\mathrm{LB}_{k} \leq \epsilon$, then stop: $x^{k}$ is a global $\epsilon$-optimal solution of problem LMP. Otherwise, set $k=k+1$, and go to Step 2 .

4.3. Convergence Analysis. In this subsection, the convergence properties of the algorithm are given.

Theorem 4. The algorithm either terminates finitely with globally $\varepsilon$-optimal solution or generates an infinite sequence 
TABLE 1: Comparison results of Examples 1-10.

\begin{tabular}{|c|c|c|c|c|c|}
\hline $\begin{array}{l}\text { Example } \\
(p, m, n) \\
\end{array}$ & Methods & Time (s) & Iter & Optimal solution & Optimal value \\
\hline \multirow{4}{*}{$\begin{array}{l}1(\min ) \\
(1,8,2)\end{array}$} & {$[13]$} & 5.0780 & 48 & $(2.0,8.0)$ & 10 \\
\hline & {$[14]$} & 0.3 & 53 & $(2.0,8.0)$ & 10 \\
\hline & {$[15]$} & 10.83 & 27 & $(2.0003,7.9999)$ & 10.0042 \\
\hline & Ours & 0.062 & 1 & $(2.0,8.0)$ & 10 \\
\hline \multirow{3}{*}{$\begin{array}{l}2(\min ) \\
(2,6,2)\end{array}$} & {$[13]$} & 0.2030 & 2 & $(0.0,4.0)$ & 3 \\
\hline & {$[16]$} & - & 3 & $(0.0,4.0)$ & 3 \\
\hline & Ours & 0.086 & 1 & $(0.0,4.0)$ & 3 \\
\hline \multirow{3}{*}{$\begin{array}{l}3(\min ) \\
(1,12,4)\end{array}$} & {$[13]$} & 0.1880 & 1 & $(1.3148,0.1396,0.0,0.4233)$ & 0.8902 \\
\hline & {$[17]$} & - & 6 & $(1.3148,0.1396,0.0,0.4233)$ & 0.8902 \\
\hline & Ours & 0.093 & 1 & $(1.3148,0.1396,0.0,0.4233)$ & 0.8902 \\
\hline \multirow{3}{*}{$\begin{array}{l}4(\min ) \\
(2,5,2)\end{array}$} & {$[8]$} & - & - & $(0.0,4.0)$ & 3 \\
\hline & {$[18]$} & - & - & $(0.0,4.0)$ & 3 \\
\hline & Ours & 0.0842 & 1 & $(0.0,4.0)$ & 3 \\
\hline \multirow{2}{*}{$\begin{array}{l}5(\min ) \\
(2,4,2)\end{array}$} & {$[19]$} & - & - & $(1.0,3.0)$ & -13 \\
\hline & Ours & 0.0150 & 1 & $(1.0,3.0)$ & -13 \\
\hline \multirow{2}{*}{$\begin{array}{l}6(\min ) \\
(2,4,2)\end{array}$} & [19] & - & - & $(1.0,4.0)$ & -22 \\
\hline & Ours & 0.0160 & 1 & $(1.0,4.0)$ & -22 \\
\hline \multirow{2}{*}{$\begin{array}{l}7(\min ) \\
(3,7,3)\end{array}$} & {$[20]$} & - & 57 & $(5.5556,1.7778,2.6667)$ & -112.754 \\
\hline & Ours & 1.5930 & 34 & $(5.5556,1.7778,2.6667)$ & -112.7531 \\
\hline \multirow{2}{*}{$\begin{array}{l}8(\max ) \\
(2,4,2)\end{array}$} & {$[21]$} & - & 13 & $(0.74984,0.74984)$ & -38.87628 \\
\hline & Ours & 0.5 & 16 & $(0.75,0.75)$ & -38.8750 \\
\hline \multirow{2}{*}{$\begin{array}{l}9(\min ) \\
(2,6,2)\end{array}$} & {$[22]$} & - & 7 & $(1.547,2.421)$ & -16.2837 \\
\hline & Ours & 0.659 & 23 & $(1.5480,2.4152)$ & -16.2893 \\
\hline \multirow{2}{*}{$\begin{array}{l}10(\min ) \\
(2,6,2)\end{array}$} & {$[22]$} & - & 29 & $(1.5549,0.7561)$ & 10.6756 \\
\hline & Ours & 0.7662 & 27 & $(1.5568,0.7545)$ & 10.6753 \\
\hline
\end{tabular}

$\left\{x^{k}\right\}$, where any accumulation point is a globally optimal solution of LMP.

Proof. If the algorithm terminates finitely, without loss of generality, assume that the algorithm terminates at the $k$ th step; by the algorithm, we have

$$
\mathrm{UB}_{k}-\mathrm{LB}_{k} \leq \varepsilon
$$

So, $x^{k}$ is a global optimal solution of the problem LMP.

If the algorithm is infinite, then an infinite sequence $\left\{x^{k}\right\}$ will be generated. Since the feasible region of LMP is bounded, the sequence $\left\{x^{k}\right\}$ must have a convergence subsequence. Without loss of generality, set $\lim _{k \rightarrow \infty} x^{k}=x^{*}$. By the algorithm, we have

$$
\lim _{k \rightarrow \infty} \mathrm{LB}_{k} \leq v
$$

Since $x^{*}$ is a feasible solution of problem LMP, $v \leq \Phi\left(x^{*}\right)$. Taken together, the following relation holds:

$$
\lim _{k \rightarrow \infty} \mathrm{LB}_{k} \leq v \leq \phi\left(x^{*}\right)
$$

On the other hand, by the algorithm and the continuity of $\phi^{l}(x)$, we have

$$
\lim _{k \rightarrow \infty} \mathrm{LB}_{k}=\lim _{k \rightarrow \infty} \phi^{l}\left(x^{k}\right)=\phi^{l}\left(x^{*}\right) .
$$

By Theorem 1, we can obtain

$$
\phi\left(x^{*}\right)=\phi^{l}\left(x^{*}\right) .
$$

Therefore, $v=\phi\left(x^{*}\right)$; that is $x^{*}$ is a global optimal solution of problem LMP.

\section{Numerical Experiments}

To verify the performance of the proposed algorithm, some numerical experiments are carried out and compared with some other methods [8,13-22]. The algorithm is compiled with Matlab 7.1 on a Pentium IV (3.06 GHZ) microcomputer. The simplex method is applied to solve the linear relaxation programming problems. In our experiments, for Examples 110, the convergence tolerance $\epsilon$ is $10^{-6}$; for Example 11, the convergence tolerance $\epsilon$ is $10^{-2}$.

The results of problems 1-10 are summarized in Table 1, where the following notations have been used in row headers: Iter is the number of algorithm iterations; Time (s) is 
TABLE 2: Comparison results of Algorithms 1 and 2 for Examples 1-10.

\begin{tabular}{|c|c|c|c|c|c|}
\hline $\begin{array}{l}\text { Example } \\
(p, m, n)\end{array}$ & Methods & Time (s) & Iter & Optimal solution & Optimal value \\
\hline \multirow{2}{*}{$\begin{array}{l}1(\min ) \\
(1,8,2)\end{array}$} & Algorithm 1 & 0.062 & 1 & $(2.0,8.0)$ & 10 \\
\hline & Algorithm 2 & 0.062 & 1 & $(2.0,8.0)$ & 10 \\
\hline \multirow{2}{*}{$\begin{array}{l}2(\min ) \\
(2,6,2)\end{array}$} & Algorithm 1 & 0.086 & 1 & $(0.0,4.0)$ & 3 \\
\hline & Algorithm 2 & 0.086 & 1 & $(0.0,4.0)$ & 3 \\
\hline \multirow{2}{*}{$\begin{array}{l}3(\min ) \\
(1,12,4)\end{array}$} & Algorithm 1 & 0.093 & 1 & $(1.3148,0.1396,0.0,0.4233)$ & 0.8902 \\
\hline & Algorithm 2 & 0.093 & 1 & $(1.3148,0.1396,0.0,0.4233)$ & 0.8902 \\
\hline \multirow{2}{*}{$\begin{array}{l}4(\min ) \\
(2,5,2)\end{array}$} & Algorithm 1 & 0.0842 & 1 & $(0.0,4.0)$ & 3 \\
\hline & Algorithm 2 & 0.0842 & 1 & $(0.0,4.0)$ & 3 \\
\hline \multirow{2}{*}{$\begin{array}{l}5(\min ) \\
(2,4,2)\end{array}$} & Algorithm 1 & 0.0150 & 1 & $(1.0,3.0)$ & -13 \\
\hline & Algorithm 2 & 0.0150 & 1 & $(1.0,3.0)$ & -13 \\
\hline \multirow{2}{*}{$\begin{array}{l}6(\min ) \\
(2,4,2)\end{array}$} & Algorithm 1 & 0.0160 & 1 & $(1.0,4.0)$ & -22 \\
\hline & Algorithm 2 & 0.0160 & 1 & $(1.0,4.0)$ & -22 \\
\hline \multirow{2}{*}{$\begin{array}{l}7(\min ) \\
(3,7,3)\end{array}$} & Algorithm 1 & 1.5930 & 34 & $(5.5556,1.7778,2.6667)$ & -112.7531 \\
\hline & Algorithm 2 & 1.9091 & 58 & $(5.5556,1.7778,2.6667)$ & -112.7531 \\
\hline \multirow{2}{*}{$\begin{array}{l}8(\max ) \\
(2,4,2)\end{array}$} & Algorithm 1 & 0.5 & 16 & $(0.75,0.75)$ & -38.8750 \\
\hline & Algorithm 2 & 1.6425 & 64 & $(0.75,0.75)$ & -38.8750 \\
\hline \multirow{2}{*}{$\begin{array}{l}9(\min ) \\
(2,6,2)\end{array}$} & Algorithm 1 & 0.659 & 23 & $(1.5480,2.4152)$ & -16.2893 \\
\hline & Algorithm 2 & 1.7235 & 39 & $(1.5480,2.4152)$ & -16.2893 \\
\hline \multirow{2}{*}{$\begin{array}{l}10(\min ) \\
(2,6,2)\end{array}$} & Algorithm 1 & 0.7662 & 27 & $(1.5568,0.7545)$ & 10.6753 \\
\hline & Algorithm 2 & 1.4862 & 68 & $(1.5480,2.4152)$ & 10.6753 \\
\hline
\end{tabular}

execution time in seconds. Except for the results of our algorithm, the results of the other eleven algorithms are taken directly from the corresponding references. In Table 1, “-” denotes the corresponding value is not available.

For problems 1-10, the efficiency of the algorithm proposed by this paper (named Algorithm 1) and the algorithm proposed by this paper but without using the pruning techniques (named Algorithm 2) is compared. The comparison results are given in Table 2 .

Example 1 (see [13-15]).

$$
\begin{array}{ll}
\min & \left(x_{1}+x_{2}\right)\left(x_{1}-x_{2}+7\right) \\
\text { s.t. } & 2 x_{1}+x_{2} \leq 14 \\
& x_{1}+x_{2} \leq 10 \\
& -4 x_{1}+x_{2} \leq 0 \\
& -2 x_{1}-x_{2} \leq-6
\end{array}
$$

$$
\begin{aligned}
& -x_{1}-2 x_{2} \leq-6, \\
& x_{1}-x_{2} \leq 3 \\
& x_{1} \geq 0, x_{2} \geq 0 .
\end{aligned}
$$

Example 2 (see $[13,16])$.

$$
\begin{array}{ll}
\min & x_{1}+\left(2 x_{1}-3 x_{2}+13\right)\left(x_{1}+x_{2}-1\right) \\
\text { s.t. } & -x_{1}+2 x_{2} \leq 8 \\
& -x_{2} \leq-3 \\
& x_{1}+2 x_{2} \leq 12, \\
& x_{1}-2 x_{2} \leq-5 \\
& x_{1} \geq 0, \quad x_{2} \geq 0 .
\end{array}
$$

Example 3 (see $[13,17])$.

$\min \left(0.813396 x_{1}+0.67440 x_{2}+0.305038 x_{3}+0.129742 x_{4}+0.217796\right)$

$$
\times\left(0.224508 x_{1}+0.063458 x_{2}+0.932230 x_{3}+0.528736 x_{4}+0.091947\right)
$$

s.t. $\quad 0.488509 x_{1}+0.063565 x_{2}+0.945686 x_{3}+0.210704 x_{4} \leq 3.562809$,

$$
\begin{aligned}
& -0.324014 x_{1}-0.501754 x_{2}-0.719204 x_{3}+0.099562 x_{4} \leq-0.052215 \\
& 0.445225 x_{1}-0.346896 x_{2}+0.637939 x_{3}-0.257623 x_{4} \leq 0.427920 \\
& -0.202821 x_{1}+0.647361 x_{2}+0.920135 x_{3}-0.983091 x_{4} \leq 0.840950
\end{aligned}
$$




$$
\begin{aligned}
& -0.886420 x_{1}-0.802444 x_{2}-0.305441 x_{3}-0.180123 x_{4} \leq-1.353686, \\
& -0.515399 x_{1}-0.424820 x_{2}+0.897498 x_{3}+0.187268 x_{4} \leq 2.137251, \\
& -0.591515 x_{1}+0.060581 x_{2}-0.427365 x_{3}+0.579388 x_{4} \leq-0.290987, \\
& 0.423524 x_{1}+0.940496 x_{2}-0.437944 x_{3}-0.742941 x_{4} \leq 0.373620, \\
& x_{1} \geq 0, x_{2} \geq 0, x_{3} \geq 0, x_{4} \geq 0 .
\end{aligned}
$$

Example 4 (see $[8,18])$.

$$
\begin{array}{ll}
\min & x_{1}+\left(x_{1}-x_{2}+5\right)\left(x_{1}+x_{2}-1\right) \\
\text { s.t. } & -2 x_{1}-3 x_{2} \leq-9 \\
& 3 x_{1}-x_{2} \leq 8 \\
& -x_{1}+2 x_{2} \leq 8 \\
& x_{1}+2 x_{2} \leq 12 \\
& x_{1} \geq 0
\end{array}
$$

Example 5 (see [19]).

$$
\begin{array}{ll}
\min & \left(x_{1}+x_{2}\right)\left(x_{1}-x_{2}\right) \\
& +\left(x_{1}+x_{2}+1\right)\left(x_{1}-x_{2}+1\right) \\
\text { s.t. } & x_{1}+2 x_{2} \leq 10 \\
& x_{1}-3 x_{2} \leq 20 \\
& 1 \leq x_{1} \leq 3 \\
& 1 \leq x_{2} \leq 3 .
\end{array}
$$

Example 6 (see [19]).

$$
\begin{array}{ll}
\min & \left(x_{1}+x_{2}\right)\left(x_{1}-x_{2}\right) \\
& +\left(x_{1}+x_{2}+2\right)\left(x_{1}\right. \\
\text { s.t. } & x_{1}+2 x_{2} \leq 20, \\
& x_{1}-3 x_{2} \leq 20, \\
& 1 \leq x_{1} \leq 4, \\
& 1 \leq x_{2} \leq 4 .
\end{array}
$$$$
+\left(x_{1}+x_{2}+2\right)\left(x_{1}-x_{2}+2\right)
$$

Example 7 (see [20]).

$$
\begin{array}{ll}
\min \quad & \left(2 x_{1}-2 x_{2}+x_{3}+2\right)\left(-2 x_{1}+3 x_{2}+x_{3}-4\right) \\
& +\left(-2 x_{1}+x_{2}+x_{3}+2\right)\left(x_{1}+x_{2}-3 x_{3}+5\right) \\
& +\left(-2 x_{1}-x_{2}+2 x_{3}+7\right)\left(4 x_{1}-x_{2}-2 x_{3}-5\right) \\
\text { s.t. } \quad x_{1} & +x_{2}+x_{3} \leq 10 \\
& x_{1}-2 x_{2}+3 x_{3} \leq 10 \\
& -2 x_{1}+2 x_{2}+3 x_{3} \leq 10
\end{array}
$$

$$
-x_{1}+2 x_{2}+3 x_{3} \geq 6,
$$$$
x_{1} \geq 1, x_{2} \geq 1, x_{3} \geq 1 \text {. }
$$

Example 8 (see [21]).

$$
\begin{array}{ll}
\max \quad & \left(-2 x_{1}+3 x_{2}-6\right)\left(3 x_{1}-5 x_{2}+3\right) \\
& +\left(4 x_{1}-5 x_{2}-7\right)\left(-3 x_{1}+3 x_{2}+4\right) \\
\text { s.t. } \quad & x_{1}+x_{2} \leq 1.5 \\
& x_{1}-x_{2} \leq 0 \\
& x_{1} \geq 0, x_{2} \geq 0
\end{array}
$$

Example 9 (see [22]).

$$
\begin{array}{ll}
\min & \left(x_{1}+2 x_{2}-2\right)\left(-2 x_{1}-x_{2}+3\right) \\
& +\left(3 x_{1}-2 x_{2}+3\right)\left(x_{1}-x_{2}-1\right) \\
\text { s.t. } & -2 x_{1}+3 x_{2} \leq 6, \\
& 4 x_{1}-5 x_{2} \leq 8, \\
& 5 x_{1}+3 x_{2} \leq 15 \\
& -4 x_{1}-3 x_{2} \leq-12, \\
& x_{1} \geq 0, x_{2} \geq 0 .
\end{array}
$$

Example 10 (see [22]).

$$
\begin{array}{ll}
\min & \left(-x_{1}+2 x_{2}-0.5\right)\left(-2 x_{1}+x_{2}+6\right) \\
& +\left(3 x_{1}-2 x_{2}+6.5\right)\left(x_{1}+x_{2}-1\right) \\
\text { s.t. } \quad & -5 x_{1}+8 x_{2} \leq 24, \\
& 5 x_{1}+8 x_{2} \leq 44, \\
& 6 x_{1}-3 x_{2} \leq 15, \\
& -4 x_{1}-5 x_{2} \leq-10, \\
& x_{1} \geq 0, \quad x_{2} \geq 0 .
\end{array}
$$

To further verify the effectiveness of Algorithm 1, a random problem with variable scale is constructed, which is defined as follows. 
Example 11.

$$
\begin{aligned}
& \min \quad f(x)=\sum_{i=1}^{p}\left(c_{i}^{T} x+d_{i}\right)\left(e_{i}^{T} x+f_{i}\right) \\
& \text { s.t. } \quad x \in D=\left\{x \mid x \in R^{n}, A x \leq b\right\},
\end{aligned}
$$

where the real elements of $c_{i}, d_{i}, e_{i}, f_{i}$ are pseudorandomly generated in the range $[-0.5,0.5]$; the real elements of $A$, $b$ are pseudorandomly generated in the range $[0.01,1]$. For Example 11, Algorithms 1 and 2 are used to solve 10 different random instances for each size and present statistics of the results. The computational results are summarized in Table 3 , where the following notations have been used in row headers: Avg.Iter is the average number of iterations; Avg.Time is the average execution time in seconds; $m$ is the number of constraints; $n$ is the number of variables.

From Table 1, it can be seen that our algorithm can determine the global optimal solution more effectively than that of $[8,13-22]$ in most cases. For Examples 8 and 9, although the number of iterations of our algorithm is more than that of the literatures [21, 22], the optimal values and optimal solutions obtained by our algorithm are better than them.

From Table 2, it can be seen that, for Examples 16, Algorithms 1 and 2 all only need one iteration to find the optimal solution; the advantage of Algorithm 1 is not reflected. However, for Examples 7-10, the performance of Algorithm 1 is better than that of Algorithm 2.

From Table 3, we can see that, for small scale problems, the advantage of Algorithm 1 is not much better than the Algorithm 2, but with the increase of the scale of the problem, the advantage of Algorithm 1 is more and more powerful. For example, when $(p, m, n)=(10,5,5)$, the average running time of Algorithms 1 and 2 is 17.0344 and 29.5778, respectively; the average iterations of Algorithms 1 and 2 are 17.6 and 30.4. However, when $(p, m, n)=(10,100,100)$, the average running time of Algorithms 1 and 2 is 42.8972 and 402.0530, respectively; the average iterations of Algorithms 1 and 2 are 56.8 and 215.3. It is clear that the efficiency of Algorithm 1 is much better than that of Algorithm 2 for large scale problems. In addition, from Table 3 , we also can see that, compared with $m$ and $n$, the impact of $p$ on our algorithm is even greater; the Avg.Time and Avg.Iter of Algorithm 1 are not increased significantly with the increase of the problem size.

The comparison results of Tables 2 and 3 show that the pruning techniques are very good at improving the convergence speed of our algorithm.

The test results show that our algorithm is both feasible and efficient.

\section{Competing Interests}

The authors declare that there is no conflict of interests regarding the publication of this paper.

\begin{tabular}{|c|c|c|c|}
\hline$(p, m, n)$ & Methods & Avg.Time & Avg.Iter \\
\hline \multirow{2}{*}{$(3,5,5)$} & Algorithm 1 & 0.8520 & 3.4 \\
\hline & Algorithm 2 & 3.8780 & 13.6 \\
\hline \multirow{2}{*}{$(3,10,10)$} & Algorithm 1 & 1.3142 & 4.2 \\
\hline & Algorithm 2 & 16.1132 & 46.8 \\
\hline \multirow{2}{*}{$(3,20,20)$} & Algorithm 1 & 23.2650 & 49.6 \\
\hline & Algorithm 2 & 32.5486 & 66.8 \\
\hline \multirow{2}{*}{$(3,100,100)$} & Algorithm 1 & 42.8972 & 62.5 \\
\hline & Algorithm 2 & 75.0706 & 32.2 \\
\hline \multirow{2}{*}{$(6,5,5)$} & Algorithm 1 & 3.7030 & 5.2 \\
\hline & Algorithm 2 & 8.6610 & 16.6 \\
\hline \multirow{2}{*}{$(6,10,10)$} & Algorithm 1 & 5.1134 & 7.8 \\
\hline & Algorithm 2 & 40.2840 & 69.8 \\
\hline \multirow{2}{*}{$(6,20,20)$} & Algorithm 1 & 12.7132 & 13.4 \\
\hline & Algorithm 2 & 100.4470 & 128.8 \\
\hline \multirow{2}{*}{$(6,100,100)$} & Algorithm 1 & 31.9760 & 25.3 \\
\hline & Algorithm 2 & 254.5428 & 183.4 \\
\hline \multirow{2}{*}{$(10,5,5)$} & Algorithm 1 & 17.0344 & 17.6 \\
\hline & Algorithm 2 & 29.5778 & 30.4 \\
\hline \multirow{2}{*}{$(10,10,10)$} & Algorithm 1 & 26.1132 & 46.8 \\
\hline & Algorithm 2 & 195.9464 & 136.8 \\
\hline \multirow{2}{*}{$(10,20,20)$} & Algorithm 1 & 32.5126 & 27.8 \\
\hline & Algorithm 2 & 249.4000 & 169.2 \\
\hline \multirow{2}{*}{$(10,100,100)$} & Algorithm 1 & 42.8972 & 56.8 \\
\hline & Algorithm 2 & 402.0530 & 215.3 \\
\hline
\end{tabular}

TABLE 3: Comparison results of Algorithms 1 and 2 for Example 11.

\section{Acknowledgments}

The research was supported by NSFC (U1404105); the Key Scientific and Technological Project of Henan Province (142102210058); the Doctoral Scientific Research Foundation of Henan Normal University (qd12103); the Youth Science Foundation of Henan Normal University (2013qk02); Henan Normal University National Research Project to Cultivate the Funded Projects (01016400105); the Henan Normal University Youth Backbone Teacher Training.

\section{References}

[1] C. D. Maranas, I. P. Androulakis, C. A. Floudas, A. J. Berger, and J. M. Mulvey, "Solving long-term financial planning problems via global optimization," Journal of Economic Dynamics and Control, vol. 21, no. 8-9, pp. 1405-1425, 1997.

[2] K. P. Bennett and O. L. Mangasarian, "Bilinear separation of two sets in $n$-space," Computational Optimization and Applications, vol. 2, no. 3, pp. 207-227, 1993.

[3] I. Quesada and I. E. Grossmann, "Alternative bounding approximations for the global optimization of various engineering design problems," in Global Optimization in Engineering Design, I. E. Grossmann, Ed., vol. 9 of Nonconvex Optimization and Its Applications, pp. 309-331, Kluwer Academic Publishers, Norwell, Mass, USA, 1996.

[4] M. C. Dorneich and N. V. Sahinidis, "Global optimization algorithms for chip layout and compaction," Engineering Optimization, vol. 25, no. 2, pp. 131-154, 1995. 
[5] J. M. Mulvey, R. J. Vanderbei, and S. A. Zenios, "Robust optimization of large-scale systems," Operations Research, vol. 43, no. 2, pp. 264-281, 1995.

[6] H. Konno, T. Kuno, and Y. Yajima, "Global minimization of a generalized convex multiplicative function," Journal of Global Optimization, vol. 4, no. 1, pp. 47-62, 1994.

[7] T. Kuno, "Solving a class of multiplicative programs with 0-1 knapsack constraints," Journal of Optimization Theory and Applications, vol. 103, no. 1, pp. 121-135, 1999.

[8] H.-S. Ryoo and N. V. Sahinidis, "Global optimization of multiplicative programs," Journal of Global Optimization, vol. 26, no. 4, pp. 387-418, 2003.

[9] P. Shen and H. Jiao, "Linearization method for a class of multiplicative programming with exponent," Applied Mathematics and Computation, vol. 183, no. 1, pp. 328-336, 2006.

[10] X.-G. Zhou and K. Wu, "A method of acceleration for a class of multiplicative programming problems with exponent," Journal of Computational and Applied Mathematics, vol. 223, no. 2, pp. 975-982, 2009.

[11] R. Horst and H. Tuy, Global Optimization: Deterministic Approaches, Springer, Berline, Germany, 2nd edition, 1993.

[12] H. P. Benson and G. M. Boger, "Outcome-space cutting-plane algorithm for linear multiplicative programming," Journal of Optimization Theory and Applications, vol. 104, no. 2, pp. 301$322,2000$.

[13] C.-F. Wang, S.-Y. Liu, and P.-P. Shen, "Global minimization of a generalized linear multiplicative programming," Applied Mathematical Modelling, vol. 36, no. 6, pp. 2446-2451, 2012.

[14] Y. L. Gao, C. X. Xu, and Y. T. Yang, "Outcome-space branch and bound algorithm for solving linear multiplicative programming," in Computational Intelligence and Security: International Conference, CIS 2005, Xian, China, December 15-19, 2005, Proceedings Part I, vol. 3801 of Lecture Notes in Computer Science, pp. 675-681, Springer, Berlin, Germany, 2005.

[15] Y. L. Gao, G. R. Wu, and W. M. Ma, "A new global optimization approach for convex multiplicative programming," Applied Mathematics and Computation, vol. 216, no. 4, pp. 1206-1218, 2010.

[16] S. Schaible and C. Sodini, "Finite algorithm for generalized linear multiplicative programming," Journal of Optimization Theory and Applications, vol. 87, no. 2, pp. 441-455, 1995.

[17] N. V. Thoai, "A global optimization approach for solving the convex multiplicative programming problem," Journal of Global Optimization, vol. 1, pp. 341-357, 1991.

[18] J. E. Falk and S. W. Palocsay, "Image space analysis of generalized fractional programs," Journal of Global Optimization, vol. 4, no. 1, pp. 63-88, 1994.

[19] H. Jiao, K. Li, and J. Wang, "An optimization algorithm for solving a class of multiplicative problems," Journal of Chemical and Pharmaceutical Research, vol. 6, no. 1, pp. 271-277, 2014.

[20] X.-G. Zhou, B.-Y. Cao, and K. Wu, "Global optimization method for linear multiplicative programming," Acta Mathematicae Applicatae Sinica, vol. 31, no. 2, pp. 325-334, 2015.

[21] X.-G. Zhou, "Global optimization of linear multiplicative programming using univariate search," in Fuzzy Information \& Engineering and Operations Research \& Management, B.-Y. Cao and H. Nasseri, Eds., vol. 211 of Advances in Intelligent Systems and Computing, pp. 51-56, 2014.

[22] X.-G. Zhou and B.-Y. Cao, "A simplicial branch and bound duality-bounds algorithm to linear multiplicative programming," Journal of Applied Mathematics, vol. 2013, Article ID 984168, 10 pages, 2013. 

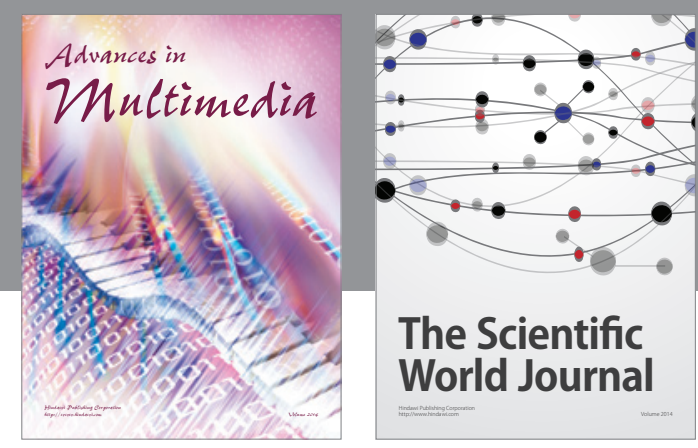

The Scientific World Journal
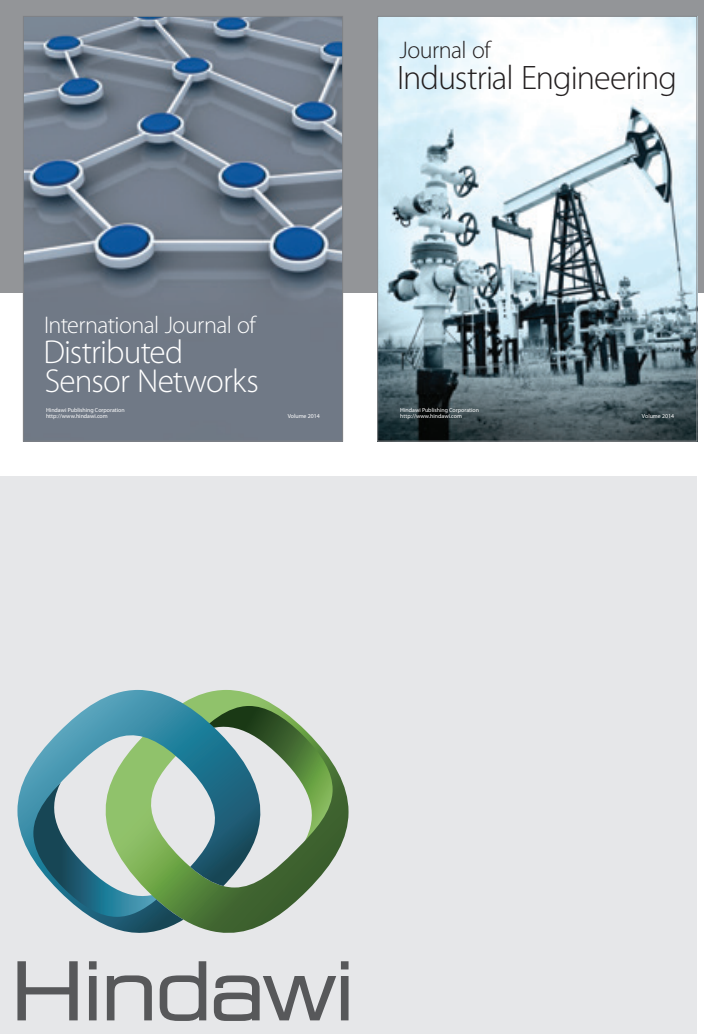

Submit your manuscripts at

http://www.hindawi.com

\section{Computer Networks} and Communications
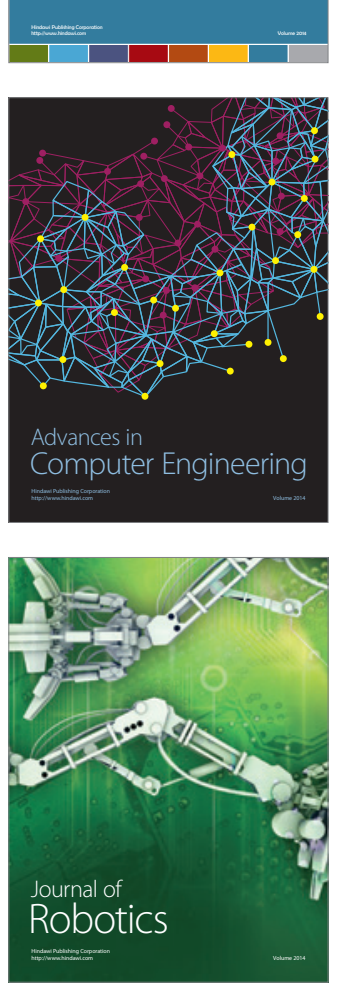
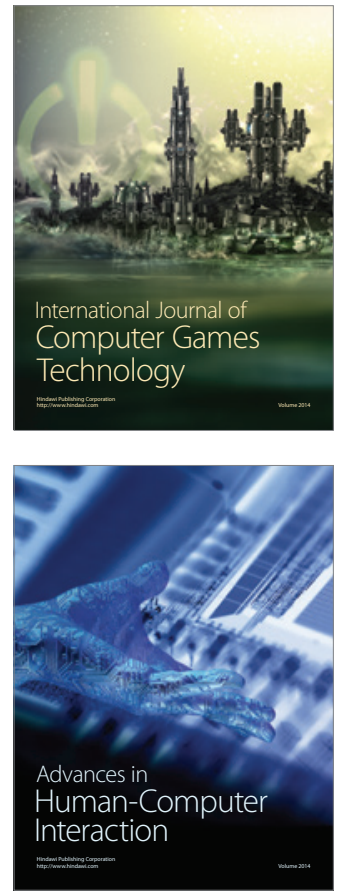
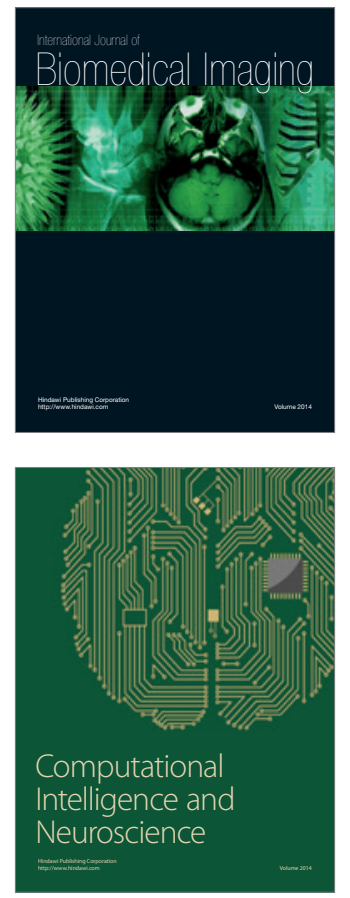
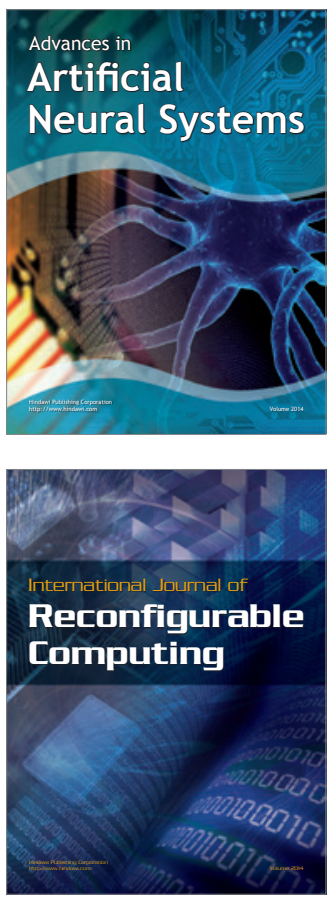
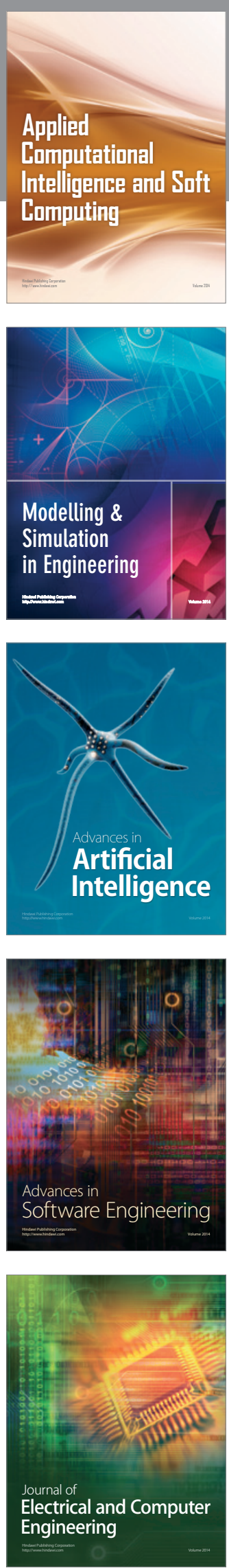